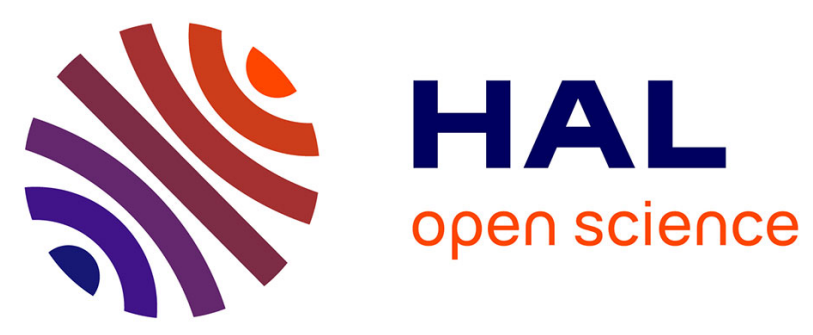

\title{
Motion capture of a pad measured with accelerometers during squeal noise in a real brake system
}

\author{
Franck Renaud, Gaël Chevallier, Jean-Luc Dion, Guillaume Taudière
}

\section{To cite this version:}

Franck Renaud, Gaël Chevallier, Jean-Luc Dion, Guillaume Taudière. Motion capture of a pad measured with accelerometers during squeal noise in a real brake system. Mechanical Systems and Signal Processing, 2012, 33, pp.155-166. 10.1016/J.YMSSP.2012.06.027 . hal-00759973

\section{HAL Id: hal-00759973 https://hal.science/hal-00759973}

Submitted on 3 Dec 2012

HAL is a multi-disciplinary open access archive for the deposit and dissemination of scientific research documents, whether they are published or not. The documents may come from teaching and research institutions in France or abroad, or from public or private research centers.
L'archive ouverte pluridisciplinaire HAL, est destinée au dépôt et à la diffusion de documents scientifiques de niveau recherche, publiés ou non, émanant des établissements d'enseignement et de recherche français ou étrangers, des laboratoires publics ou privés. 


\title{
Motion capture of a pad measured with accelerometers during squeal noise in a real brake system
}

\author{
Franck Renaud ${ }^{\mathrm{a}}$, Gaël Chevallier ${ }^{\mathrm{a}}$, Jean-Luc Dion ${ }^{\mathrm{a}}$, Guillaume Taudière ${ }^{\mathrm{b}}$ \\ ${ }^{a}$ LISMMA-EA2336 - Institut Superieur de Mecanique de Paris - 3 rue Fernand Hainaut - 93400 SAINT OUEN - FRANCE \\ ${ }^{b}$ BOSCH - Chassis Systems Brakes - 126 Rue de Stalingrad - 93700 DRANCY - FRANCE
}

\begin{abstract}
The operating deflection shape of a pad during squeal noise is measured on a real brake system with three-axis accelerometers. A time-frequency analysis is performed that highlights the dependency of squeal on the hydraulic pressure of the system and shows that squeal occurs simultaneously with harmonic components. The operating deflection shape of this pad before and during squeal is then visualized using interpolation, showing the predominance of bending motion. Finally, the pad motions observed are compared to the real modal basis of a detailed Finite Element model.
\end{abstract}

Keywords: Squeal, brake system, motion capture, operating deflection shape

\section{Introduction}

Many studies have been performed in view to modeling the squeal noise phenomenon. Two different analysis methodologies using the finite element method are avalaible for predicting disc brake squeal, namely complex eigenvalue analysis and dynamic transient analysis. However, the literature is less abundant regarding experimental work. Some of the authors of interesting works on the topic have chosen to work on simplified test rigs. Giannini et al. [1] designed a simplified squeal bench with a caliper composed of beams on which brake pads were fixed. They measured the operational deflections with a laser vibrometer. The squeal noises observed were obtained at low speed - 5 to 30 $\mathrm{rpm}$ - and exhibited harmonics. The frequency of the squeal was dependent on the normal load and the angle of the leading edge of the pad. During squeal occurrences, out of plane motions were in phase quadrature with in plane movements. The squeal noises occurred at frequencies close to those of the normal modes of the system, including modes involving the in-plane movement of the pads. Giannini showed that squeal does not require an established stick-slip limit cycle. It is usually attributed to the coupling of brake normal modes due to friction between the pads and the disc (see Giannini et al. [2]). However, in another study Giannini and Massy [3] observed modes of a rotating disc that could not be predicted with a linear model. They concluded that nonlinear aspects of the contact between disc and pad must be taken into account. Some of the conclusions of these works are well summarized in the paper by Akay, [4]. More recently, Butlin et al., [5] proposed a "systematic experimental study of squeal initiation" using a simplified test bench designed by Duffour et al. [6]. As with the work cited previously, the system consisted of two parts with a sliding contact at a single point. Numerous parameters such as contact pressure and sliding velocity were studied. The authors also provided an original study on the repeatability of the phenomenon.

All these works have developed simplified test benches to make interpreting the phenomena observed easier and facilitate updating of the models. This goal has now been partially achieved. However, some phenomena are specific to industrial brakes, in particular the size of a real plate provides a large contact area, leading to effects very different from those that can be seen with a point contact. AbuBakar and Ouyang [7] pointed out that due to the topography

Email addresses: franck.renaud@supmeca.fr (Franck Renaud), gael.chevallier@supmeca.fr (Gaël Chevallier), jeanluc.dion@supmeca.fr (Jean-Luc Dion), guillaume.taudiere@fr.bosch.com (Guillaume Taudière) 
of real brake pads, the effective contact area of a real brake pad is small in comparison to the whole area. Thus pad topography must be measured and taken into account in the geometric definition to predict disc brake squeal using complex eigenvalue analysis (cf. AbuBakar and Ouyang [8]). Furthermore, complex eigenvalue analysis allows finding all the unstable frequencies in one run, but only a few are observed in experiments. Dynamic transient analysis is able to predict true unstable frequencies but it is very time consuming. AbuBakar and Ouyang [9] have shown that care must be taken when formulating the contact model for these two different analysis methodologies.

The energy of the squeal stems from the friction between the pads and disc, thus the pads play an important role in the squeal noise phenomena. For example, chamfered and slotted pads can reduce occurrences of squeal (cf. Saw et al. [10]). Liu et al. [11] have described a method for designing chamfers based on knowledge of free-free pad modes with a frequency close to that of squeal noise. In order to improve realistic models and obtain better understanding of the phenomenon, it is necessary to capture detailed information on the operating deflection shape of the pads. Although many studies, such as that of Lorang et al. [12], compare unstable frequencies between models and experiments, few have focused on the operating deflection shapes of realistic braking devices. The first technique that comes to mind is the laser velocimetry technique, but it only allows visualizing stationary motion. Unfortunately, squeal is a more transient phenomenon than a stationary one. Fieldhouse and Newcomb [13] used a laser technique, namely doublepulse holography, to visualize the motion of a disc brake system. They observed that the trailing edge of the finger pad exhibited a swashplate type motion while the leading edge was in pure bending. The double-pulse holography technique requires a long time to set up before observation. Moreover, it does not allow observation of the operating deflection shape of hidden parts, such as the piston pads, a problem overcome by using three-axis accelerometers that allow observing the transient motion of such parts.

Piston pad motion is observed in a real brake system immediately before squeal noise occurrence and during squeal. These motions are compared together and with the normal modes of a Finite Element model.

\section{Test bench description and time-frequency analysis}

The experimental set-up consisted of a real brake system without knuckle and with a disc rotated by an electric motor (see Figures 1 and 2). The disc was driven at $2.62 \mathrm{~Hz}$ with a controller operating at a sampling frequency of $300 \mathrm{~Hz}$. During these tests, the inner ${ }^{1}$ pad was instrumented with six three-axis accelerometers. As shown in Figure 3 , the sensors were all stuck on the backplate ${ }^{2}$ with Cyanolite glue and placed as far as possible from each other: two at the leading edge (accelerometers 1 and 6), two at mid-length (accelerometers 2 and 5) and two at the trailing edge (accelerometers 3 and 4), see Figure 3. The accelerometers weighed between $3 \mathrm{~g}$ and $10 \mathrm{~g}$ and the pad weighed about $500 \mathrm{~g}$. Their position on the backplate allows describing bending and torsion motions. The sampling frequency of the data acquisition hardware was set at $f_{S}=16384 \mathrm{~Hz}$. An anti-aliasing filter was used, thus the upper frequency limit for frequency analysis was $6400 \mathrm{~Hz}$.

Here we present the results of a test at a rotation speed of about $157 \mathrm{rpm}$ (about 2.6Hz). The leading edge of the pad is on the left side and the trailing edge on the right, see Figure 3. The squeal noise was obtained by changing the brake pressure manually from 0 to 10 bars. An approximation of the brake pressure level was displayed on the control monitor but it was impossible to acquire this signal during the test. The unstable vibration leading to squeal occurred at frequencies between 1500 and $2000 \mathrm{~Hz}$, depending on the brake pressure.

\subsection{Sequence of squeal occurrence}

The top of Figure 4 shows the acceleration versus time measured by the sensor number 6 along the z-axis (see numbering in Figure 3). The Discrete Short Time Fourier Transform (DSTFT) of the same signal is presented at the bottom of Figure 4. It provides the main frequency component of a signal at a given time and was computed by using a sliding Hamming window in order to improve detection capabilities ${ }^{3}$. Each window consisted of 1024 points, corre-

\footnotetext{
${ }^{1}$ The inner pad was the pad between the disc and the vehicle.

${ }^{2}$ The backplate is the metal plate behind the lining.

${ }^{3}$ The Matlab ${ }^{\circledR}$ function "spectrogram" was used.
} 


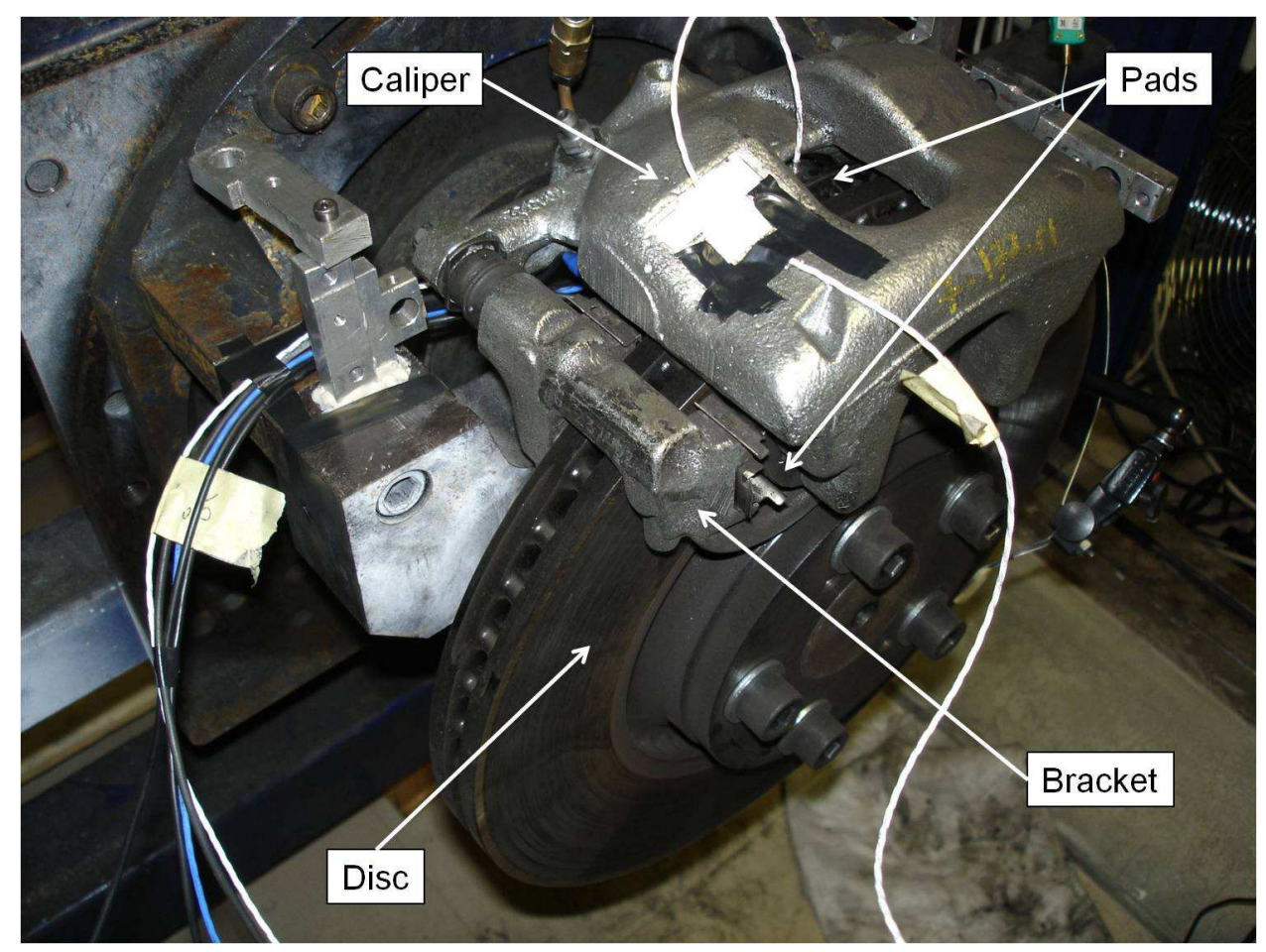

Figure 1: Test bench.

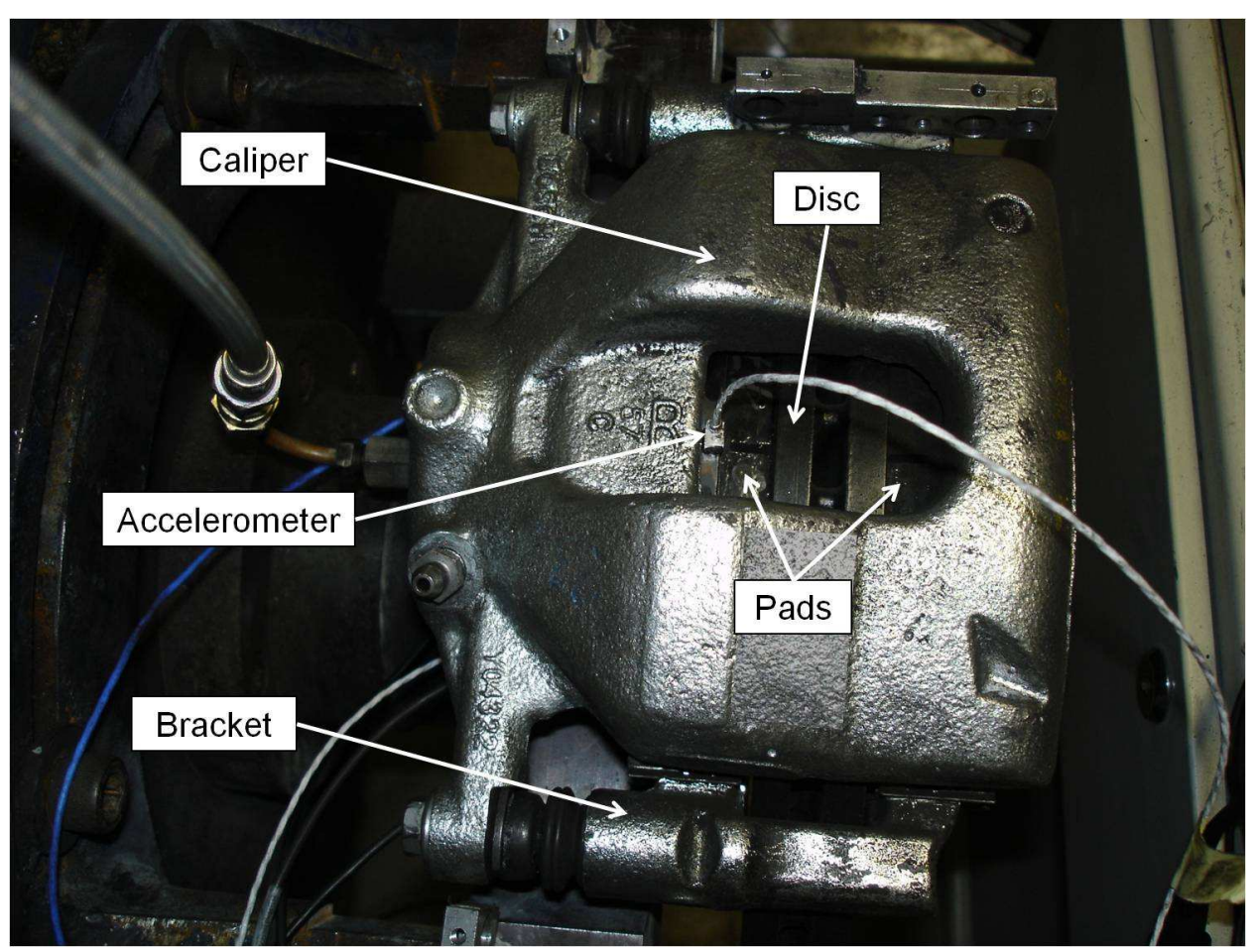

Figure 2: Test bench. 


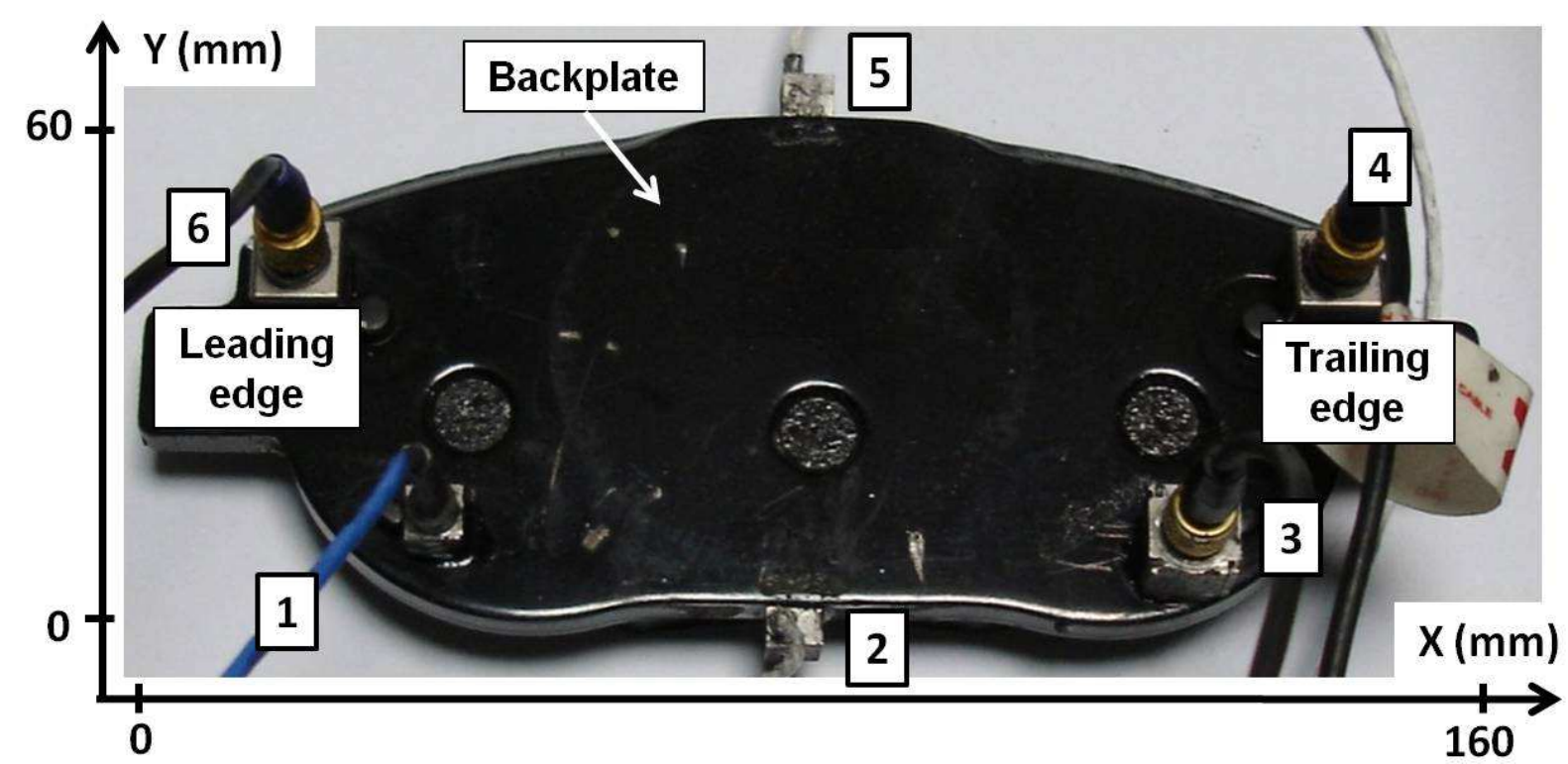

Figure 3: Backplate of the piston pad with 6 three-axis accelerometers.

sponding to a time period of $0.0625 \mathrm{~s}$ with an overlap of $50 \%$ between successive windows. The color scale represents the magnitude of the acceleration in logarithmic scale. The approximative brake pressure level is reported in Figure 4.

The time signal shown in Figure 4 can be divided into eight time periods:

1. From 0 to 2.8s: The brake pressure is nil but the pad scrapes the disc lightly, exciting the brake at all frequencies. Disc thickness variations generate acceleration variations as a function of the revolutions of the disc.

2. From 2.8 to $3.7 \mathrm{~s}$ : The brake pressure is set. All frequencies above $3000 \mathrm{~Hz}$ are excited. Squeal has not yet occurred although there is already a small frequency component around $1515 \mathrm{~Hz}$.

3. From 3.7 to $4.05 \mathrm{~s}$ : At $3.7 \mathrm{~s}$, the acceleration signal starts to diverge. At $4.05 \mathrm{~s}$ the brake pressure reaches 3 bars and the squeal noise occurs with a strong $1515 \mathrm{~Hz}$ component in the DSTFT.

4. From 4.05 to 7.9 s: The pressure increases from 3 to 6 bars. The squeal noise continues up to 7.9 s. It is composed of three main frequencies, a fundamental (H1) evolving from 1500 to $1900 \mathrm{~Hz}$ and harmonics (H2 and H3).

5. From 7.9 to $8.3 \mathrm{~s}$ : The pressure increases from 6 to 7 bars and the squeal noise disappears along with the harmonics. Although it is no longer heard, the fundamental frequency of the squeal is still latent with a lower level of magnitude.

6. From 8.3 to 8.9 s: The pressure increases from 7 to 8 bars and the squeal noise reappears. Simultaneously the harmonics reappear with a lower level than previously.

7. From 8.9 to 13.4s: The pressure increases and the squeal disappears again. This time, the frequency of the fundamental latent squeal disappears gradually. 
8. From 13.4 to $14 \mathrm{~s}$ : The pressure is released at the end of the test and the pad scrapes the disc lightly, as it did at the beginning.
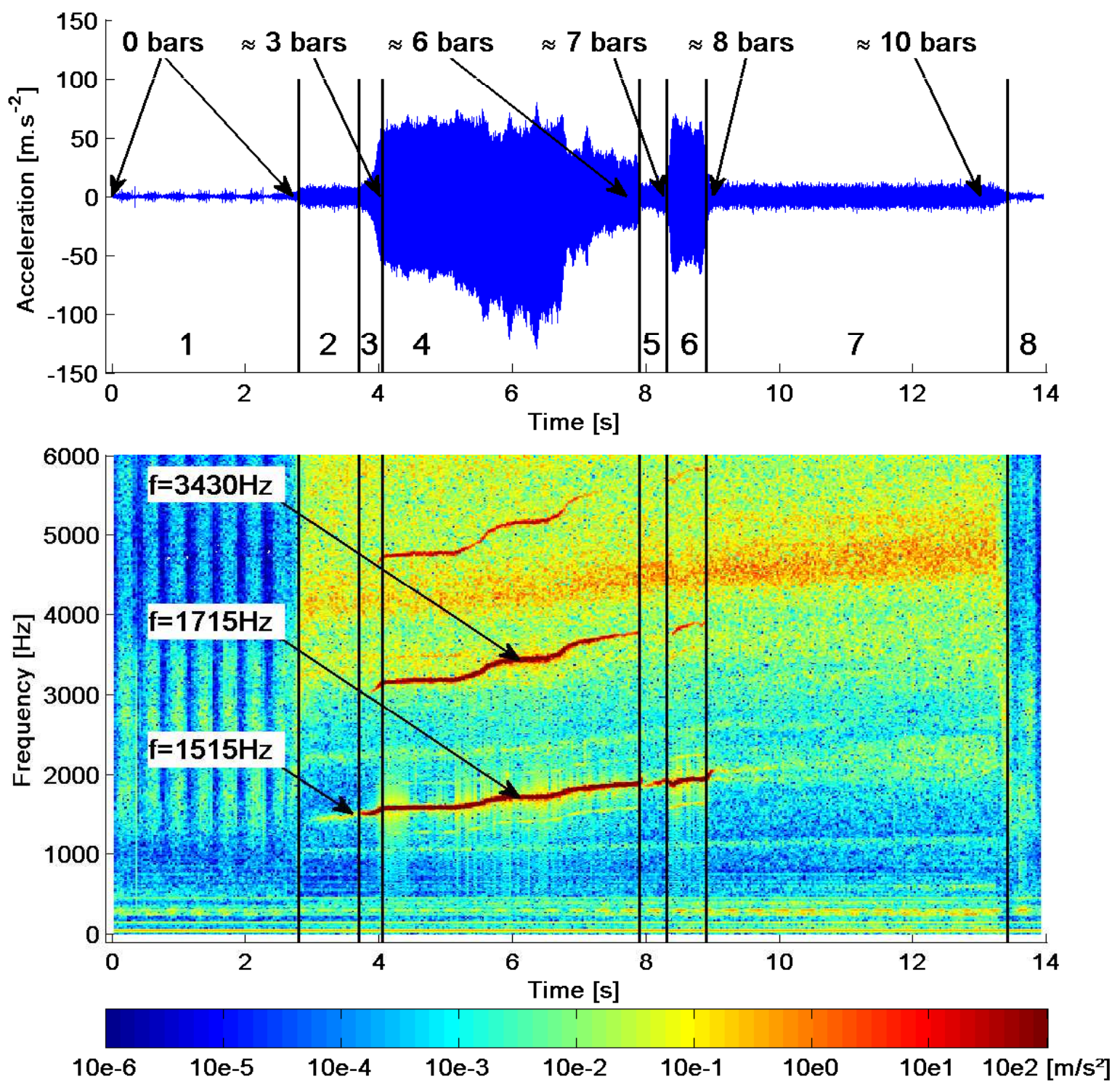

Figure 4: The acceleration versus time measured by the sensor number 6 along the $\mathrm{z}$-axis at the leading edge is shown at the top whereas the Discrete Short Time Fourier Transform (DSTFT) of the same signal is shown at the bottom. The color scale represents the magnitude of the acceleration in logarithmic scale. The signal can be divided into eight time periods described in section 2.1 . The evolution of the brake pressure is indicated.

The presence of harmonics in the accelerometer signal during squeal indicates the existence of nonlinearities. The authors would like to point out that in this test, the stick-slip phenomenon was not responsible for the squeal noise. The maximum acceleration was read by sensor 1 and remained below $200 \mathrm{~m} . \mathrm{s}^{-2}$ for a frequency higher than 
$1700 \mathrm{~Hz}$. The vibrating velocity of the pad backplate was $V_{\text {pad }}<200 /(2 \pi \times 1700)=0.019 \mathrm{~m} \cdot \mathrm{s}^{-1}$. However, the acceleration was measured on the backplate and not on the contact area, thus the velocity of the pad on the contact area was slightly higher. Considering a rigid disc, it can be estimated that the velocity of the pad on the contact area was about $V_{\mathrm{pad}} \simeq V_{x}+E_{\mathrm{pad}} / G_{\mathrm{pad}} \times V_{z} \simeq 0.019 \times\left(1+E_{\mathrm{pad}} / G_{\mathrm{pad}}\right) \simeq 4 \times 0.019 \mathrm{~m} . \mathrm{s}^{-1}$ with $E_{\mathrm{pad}}$ its Young modulus and $G_{\text {pad }}$ its shear modulus. Considering the inner radius of the disc equal to $10 \mathrm{~cm}$, the speed of the disc was $V_{\text {disc }}=0.1 \times 2 \pi \times 2.62=1.65 \mathrm{~m} \cdot \mathrm{s}^{-1}$. The speed of the pad along the $\mathrm{x}$-axis was approximately twenty times lower than the tangential velocity of the area of the disc in contact with the pad. The relative speeds between pad and disc never cancelled each other, thus the pad could not stick to the disc and the occurrence of the stick-slip phenomenon was not possible.

It should be highlighted that the squeal frequency increased with brake pressure during the fourth time period. One of several possible explanations is that contact stiffness increases with brake pressure, meaning that the contact stiffness between the piston and the pad or between the pad and the disc would be non-linear.

\subsection{Harmonic rate analysis}

Figure 4 show that the magnitude of acceleration at the second harmonic, $\mathrm{H}_{2}$ is about ten times stronger than the magnitude of acceleration at the third harmonic, $H_{3}$. Thus only the magnitude, $\mathrm{H}_{2}$, of the second harmonic is compared to the magnitude of acceleration, $H_{1}$, at the fundamental frequency $f_{\text {fundamental }}$.

The harmonic rates $H_{2} / H_{1}$ are computed between $t=3.9 \mathrm{~s}$ and $t=7.9 \mathrm{~s}$, using Equation (1), with a sliding Hanning window, $w_{N}[k]$, applied to time signals. The width of the windows is $0.1 \mathrm{~s}$ and the overlap is $75 \%$, with $N$ being the number of samples in the window.

$$
\frac{H_{2}}{H_{1}}[n]=\frac{\left|\sum_{k=0}^{N-1} w_{N}[k-n] \times \operatorname{acc}[k] \times e^{-4 i \pi k m}\right|}{\left|\sum_{k=0}^{N-1} w_{N}[k-N] \times \operatorname{acc}[k] \times e^{-2 i \pi k m}\right|} \text { with } m=f_{\text {fundamental }} / f_{S}
$$

Figure 5 shows the evolution of harmonic rates $H_{2} / H_{1}$ versus time during the occurrence of squeal in the fourth time period (from 4.05 to $7.9 \mathrm{~s}$ ). A zoom of the acceleration measured by sensor No. 6 along the z-axis is shown at the top of Figure 5. Below are represented the evolutions of harmonic rates classified according to the direction of observation, for sensors 6 and 4, at the leading edge and at the trailing edge.

These harmonic rates are strong according to the z-axis, the normal direction to the disc, and to the y-axis, the radial direction. On the contrary, the tangential component, the $\mathrm{x}$-axis, does not have strong harmonic components. This interesting difference between directions as well as the cause of the harmonics have not yet been explained. The investigation of relative displacements between the pad and the other mechanical parts should provide an answer in future work. However, the lack of harmonics along the $\mathrm{x}$-axis may indicate that the behavior of the pad abutment contacts stays fairly linear in this direction. The authors would like to point out that non-linearities along the z-axis induce non-linear friction forces in the xy-plane due to friction.

The effective contact surface between pad and disc has been proved to be small in comparison to the nominal surface of the pad (cf. AbuBakar and Ouyang [7]). Moreover the effective contact surface is divided into several local contacts. These local contacts can be modelled classically on the basis of Hertz's theory according to which the effective contact surface depends on the normal force. This dependence leads to a non linear contact stiffness. The compressibility of the whole pad was measured (cf. Figure 6) and seemed to obey an exponential law. This non linear behavior may be due to the pad material or to the contact. Thus the non-linear stiffness between the pad and the disc is one possible explanation for the harmonics observed in the z-axis and in the y-axis due to friction. 

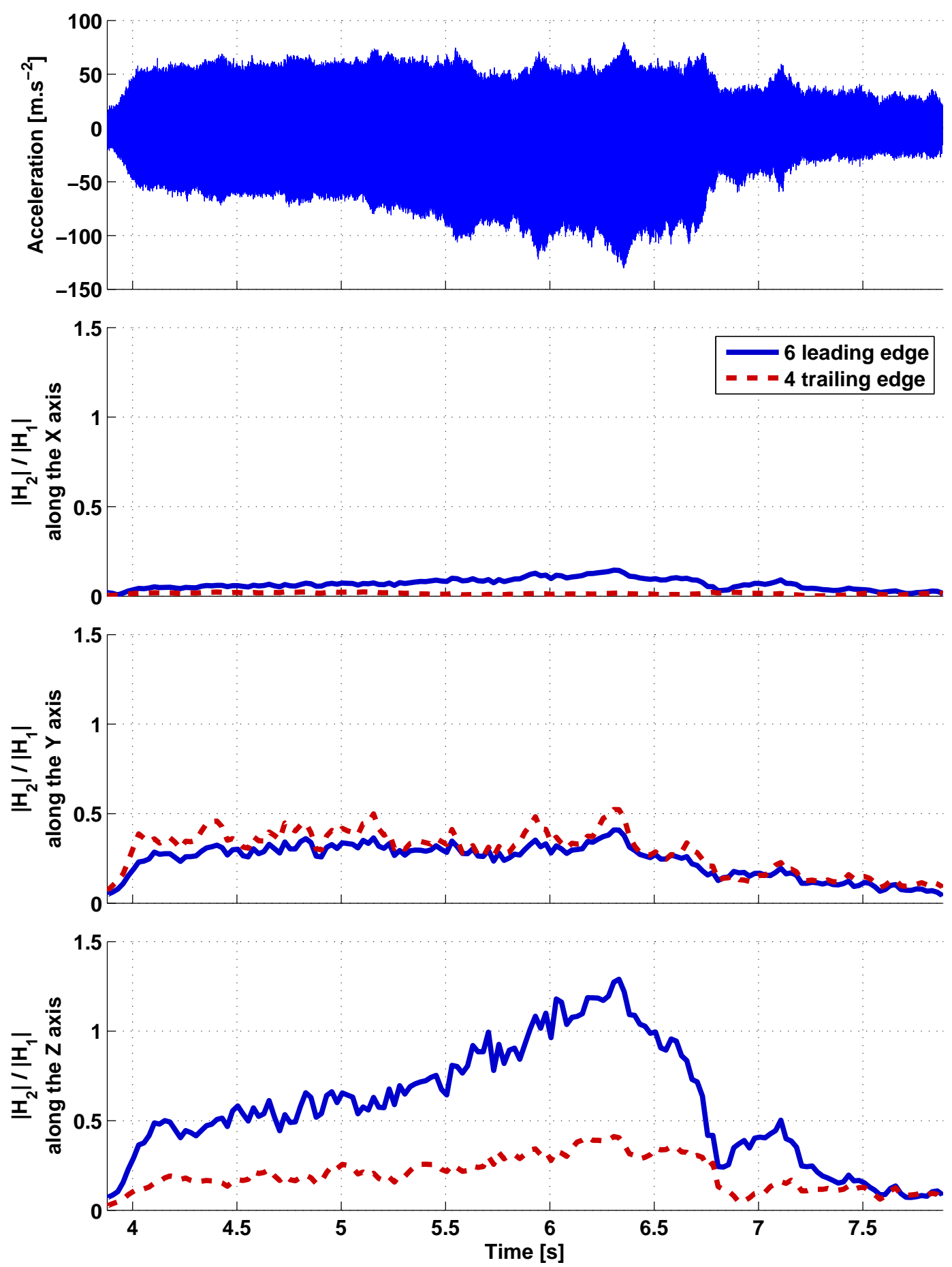

Figure 5: Evolution of harmonic rates $H_{2} / H_{1}$ versus time of accelerometer measurements during the squeal occurence of the fourth time period (from 3.9 to $7.9 \mathrm{~s}$ ). $H_{1}$ is the acceleration magnitude at the fundamental frequency and $H_{2}$ is the acceleration magnitude at the frequency of the second harmonic. A zoom of the acceleration measured by the sensor No. 6 along the z-axis is represented at the top. Below are represented the evolutions of the harmonic rates for sensors 4 and 6 classified according to the direction of observation. 


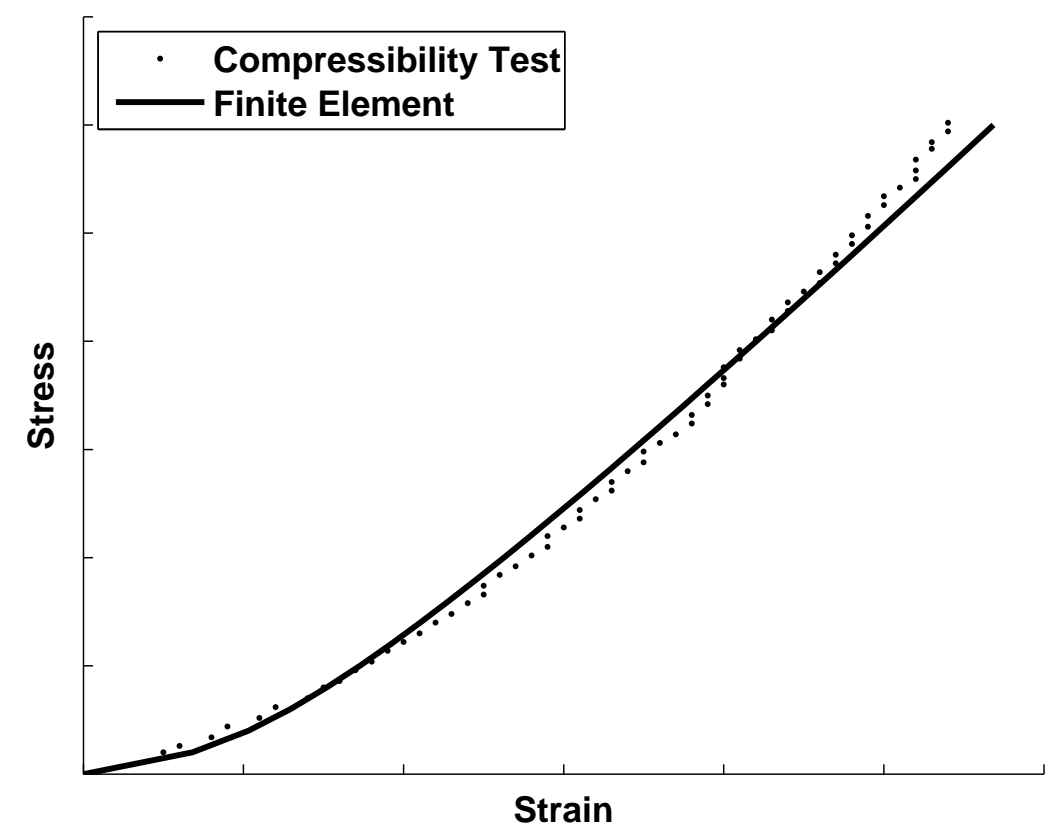

Figure 6: The measured compressibility of the whole pad (dots) and the compressibility of the whole pad computed using a Finite Element model (solid line). An exponential law is considered in the FE model. The axes are not filled because the values are confidential.

\section{Pad operating deflection shape}

\subsection{Before the squeal noise}

The motions of the backplate pad can be interpolated using the six three-axis accelerometers stuck on it. The interpolation is computed by Finite Element interpolation (cf. Dhatt et al. [14]). The element is a quadrilateral composed of six nodal variables with a quadratic interpolation along the $\mathrm{x}$-axis and a linear interpolation along the y-axis (cf. Figure 7).

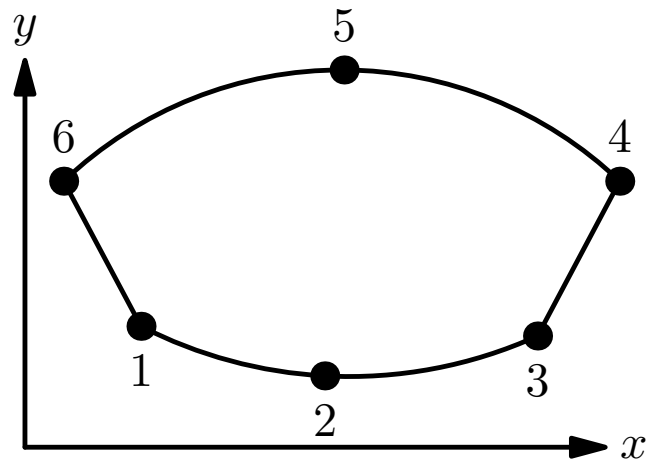

Figure 7: Quadrilateral element composed of six nodal variables with a quadratic interpolation along the $\mathrm{x}$-axis and a linear interpolation along the $y$-axis.
The polynomial basis is $\langle P(x, y)\rangle=\left\langle 1, x, y, x y, x^{2}, x^{2} y\right\rangle$. Displacements are interpolated using the following equation: $u(x, y)=$ $\langle N(x, y)\rangle\left\{u_{n}\right\}$. Here $\left\{u_{n}\right\}$ is the vector of the sensor displacements computed by integrating the acceleration measurements. Interpolation functions are computed using the following equation: $\langle N(x, y)\rangle=\langle P(x, y)\rangle\left[P_{n}\right]^{-1}$. Here, $\left[P_{n}\right]$ is computed by evaluating the polynomial basis at each sensor position (cf. Eq. (2)).

$$
\left[P_{n}\right]=\left[\begin{array}{cccccc}
1 & x_{1} & y_{1} & x_{1} y_{1} & x_{1}^{2} & x_{1}^{2} y_{1} \\
1 & x_{2} & y_{2} & x_{2} y_{2} & x_{2}^{2} & x_{2}^{2} y_{2} \\
1 & x_{3} & y_{3} & x_{3} y_{3} & x_{3}^{2} & x_{3}^{2} y_{3} \\
1 & x_{4} & y_{4} & x_{4} y_{4} & x_{4}^{2} & x_{4}^{2} y_{4} \\
1 & x_{5} & y_{5} & x_{5} y_{5} & x_{5}^{2} & x_{5}^{2} y_{5} \\
1 & x_{6} & y_{6} & x_{6} y_{6} & x_{6}^{2} & x_{6}^{2} y_{6}
\end{array}\right]
$$

Let us focus on the pad motion immediately before the onset of squeal, i.e. between $t=3.6 \mathrm{~s}$ and $t=3.8 \mathrm{~s}$. According to the DSTFT of Figure 4, the acceleration signal is mainly composed of noise above $3000 \mathrm{~Hz}$ plus a $1515 \mathrm{~Hz}$ frequential component. Since this $1515 \mathrm{~Hz}$ frequential component leads to squeal noise in the fourth time period, it is clearly due to an unstable mode of the brake system. The frequential components of the acceleration 
measurements, $A c c_{\text {pad }}$, at $1515 \mathrm{~Hz}$ are extracted using Equation (3) by applying a Hanning window between $t=3.6 \mathrm{~s}$ and $t=3.8 \mathrm{~s}$. Then, they are integrated to compute the vector of sensor displacements : $\left\{u_{n}\right\}=-A c c_{\mathrm{pad}} /\left(2 \pi m f_{S}\right)^{2}$. The motion of the pad at $1515 \mathrm{~Hz}$ is shown in Figure 8.

$$
A c c_{\mathrm{pad}}=\sum_{k=0}^{N-1} w_{N}[k] \times a c c[k] \times e^{-2 i \pi k m} \text { with } m=1515 / f_{S}
$$

The pad motion shown in Figure 8 is a bending motion. Moreover, the acceleration spectrum values at $1515 \mathrm{~Hz}$ obtained thanks to Equation (3) are complex. This indicates movement propagated by the pad which can be seen on the video available in the online version of the paper (cf. Figure 8).

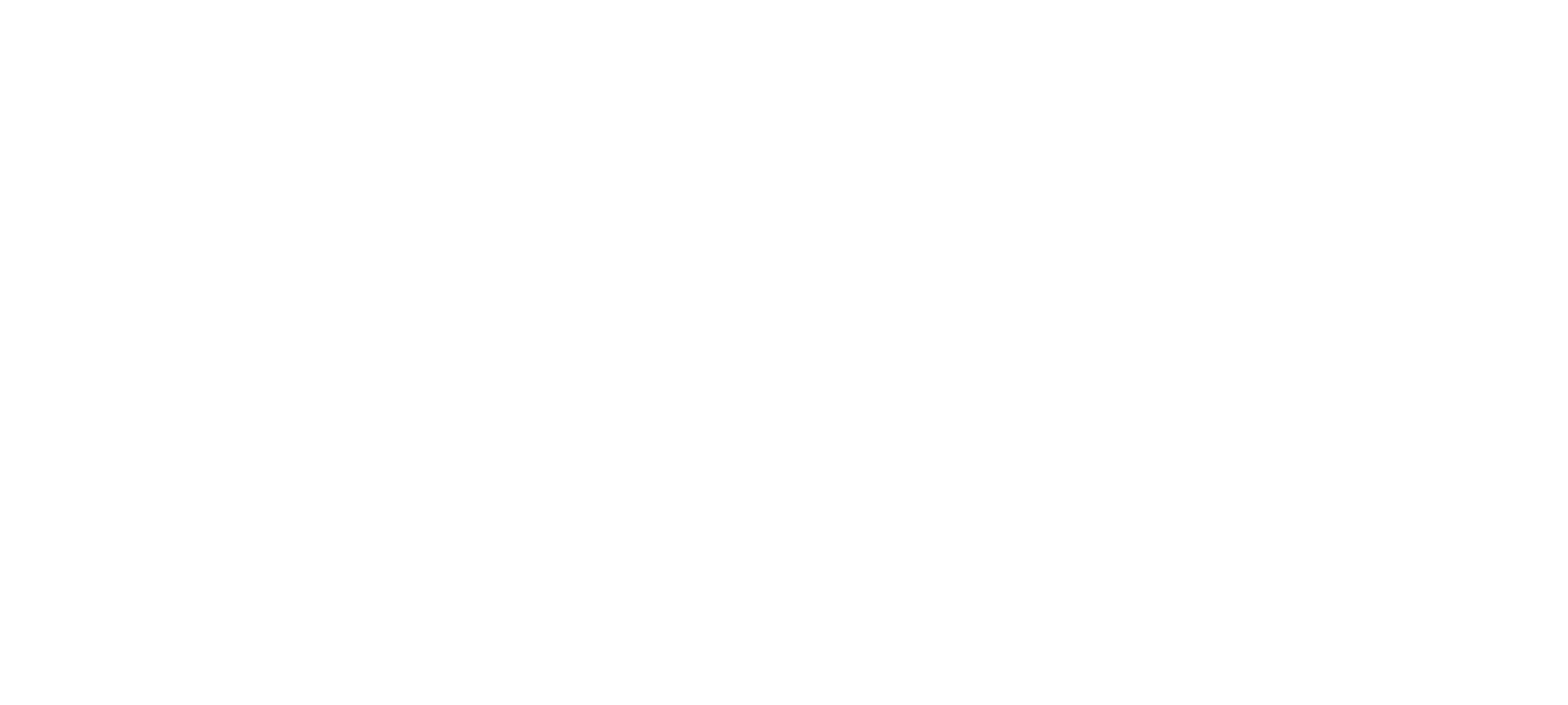

Figure 8: Motion of the pad backplate immediately before the occurrence of squeal noise. In order to see the motion of the pad between $t=3.6 \mathrm{~s}$ and $t=3.8 \mathrm{~s}$ at the fundamental frequency $(1515 \mathrm{~Hz})$, the acceleration measurements were extracted by applying Equation $(3)$. The color scale represents the magnitude of displacement in meters along the $\mathrm{z}$-axis.

\subsection{During the squeal noise}

Let us focus on the pad motion during the squeal noise, between $t=6.0 \mathrm{~s}$ and $t=6.2 \mathrm{~s}$. According to the DSTFT of Figure 4, the acceleration signal is mainly composed of three frequential components, the fundamental at $1715 \mathrm{~Hz}$ and two harmonics at $3430 \mathrm{~Hz}$ and $5145 \mathrm{~Hz}$. Thus the pad motion during squeal occurrence is the sum of motions at the fundamental frequency and the harmonics frequencies. The maximum displacement magnitude occurs at the fundamental frequency. Thus the whole motion is mainly represented by the motion at the fundamental frequency. As done before in section 3.1, the frequential components of the acceleration measurements at $1715 \mathrm{~Hz}$ and $3430 \mathrm{~Hz}$ are extracted using Equation (3), now with $m=1715 / f_{S}$ for the fundamental and $m=3430 / f_{S}$ for the second harmonic. They are then integrated to compute the vector of sensor motions: $\left\{u_{n}\right\}$. These pad motions are both represented in Figure 9 and 10.

$$
\operatorname{MAC}_{i j}=\frac{\left|{ }^{t} \varphi_{i} \overline{\psi_{j}}\right|^{2}}{{ }^{t} \varphi_{i} \overline{\varphi_{i}}{ }^{t} \psi_{j} \overline{\psi_{j}}}
$$

The Modal Assurance Criterion (MAC) defined by Equation (4) is a measurement of the degree of linearity between two vectors $\varphi_{i}$ and $\psi_{j}$ (cf. Allemang [15]). On the one hand, the fundamental pad motion during the squeal (cf. Figure 9) is the same bending motion as immediately before the squeal (cf. Figure 8). The MAC between these two 


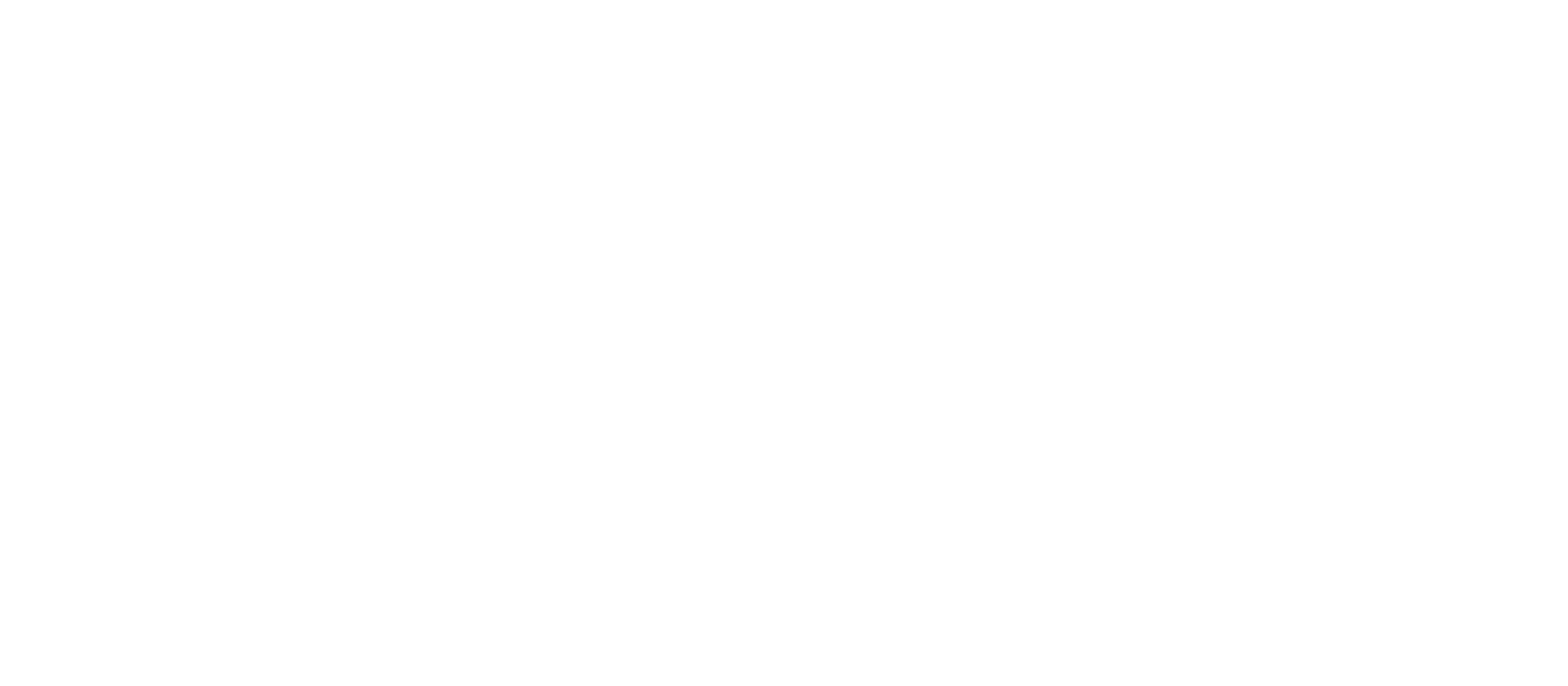

Figure 9: Motion of the pad backplate during the occurrence of squeal noise. In order to see the motion of the pad between $t=6.0 \mathrm{~s}$ and $t=6.2 \mathrm{~s}$ at the fundamental frequency $(1715 \mathrm{~Hz})$, the acceleration measurements were extracted using Equation (3). The color scale represents the magnitude of displacement in meters along the z-axis.

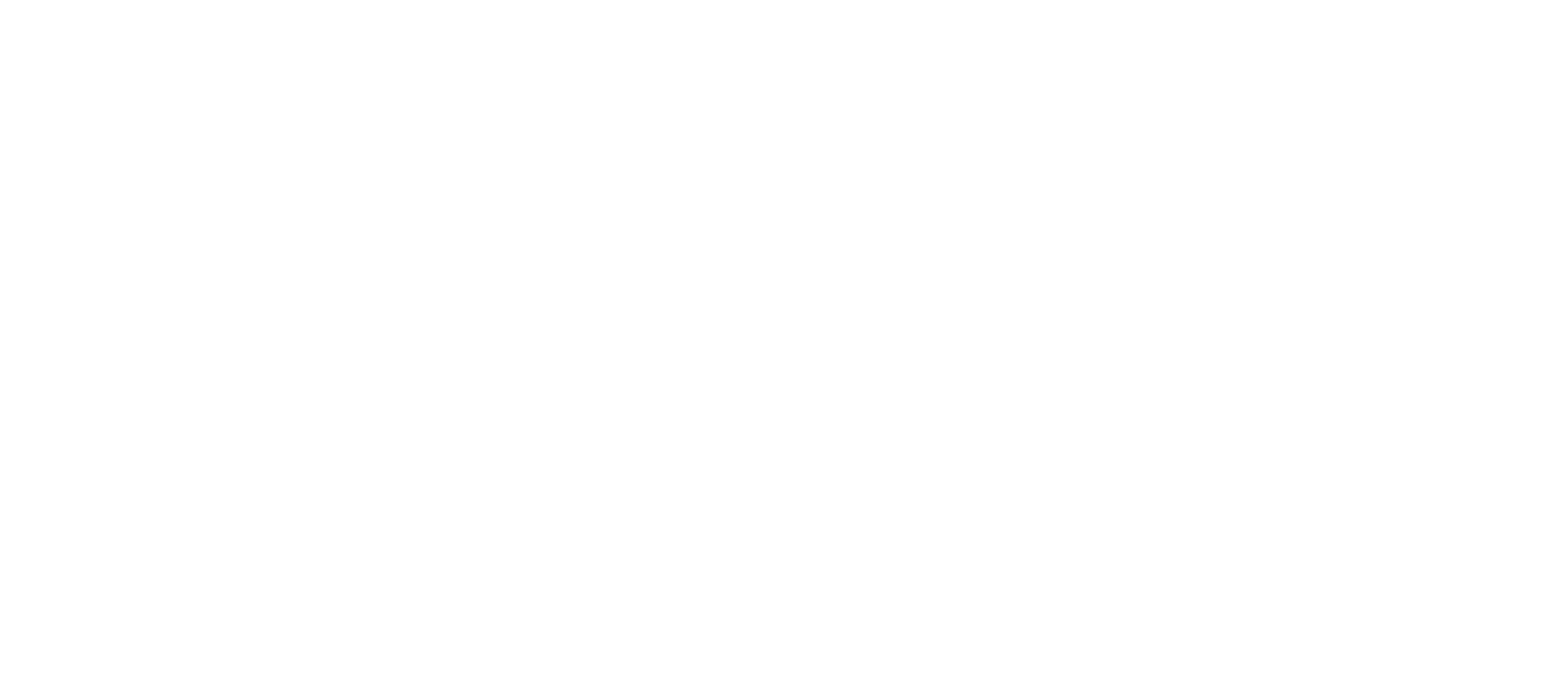

Figure 10: Motion of the pad backplate during the occurrence of squeal noise . In order to see the motion of the pad between $t=6.0 \mathrm{~s}$ and $t=6.2 \mathrm{~s}$, at the second harmonic $(3430 \mathrm{~Hz})$, the acceleration measurements were extracted using Equation (3). The color scale represents the magnitude of displacement in meters along the $\mathrm{z}$-axis.

motions is $88.6 \%$. However, during the occurrence of squeal, the amplitude of the motion was a hundred times higher. Moreover, by separating the direction of observation, the authors obtained a MAC value of $99.17 \%$ for the $\mathrm{x}$-axis, $90.7 \%$ for the $\mathrm{y}$-axis and $96.3 \%$ for the $\mathrm{z}$-axis. On the other hand, the second harmonic pad motion between $t=6.0 \mathrm{~s}$ and $t=6.2 \mathrm{~s}$ was composed of a torsion plus a bending motion, mostly involving the leading edge (cf. Figure 10). As seen previously, the acceleration spectrum values at $1715 \mathrm{~Hz}$ and $3430 \mathrm{~Hz}$ obtained using Equation (3) are complex. 
This denotes a propagation movement of the pad. This can be seen on the videos available in the online version of the paper (cf. Figure 9 and Figure 10).

\section{Discussion on the consequences on the Finite Element tool}

It has now become commonplace to use Finite Element (FE) approaches for squeal prediction. There are typically two different methodologies, namely, complex eigenvalue analysis and dynamic transient analysis (cf. AbuBakar and Ouyang [9]). Both are very time-consuming when used for a very large FE model, i.e. with more than 100,000 degrees of freedom. Thus a classical approach is to reduce the size of an FE model by projection on its modal basis. Modal bases are computed using real eigenvalue analysis and are considered to represent the behaviour of FE models well. Here, the authors investigate and discuss the correlation between the modal basis of a detailed FE model and the pad motions observed during the experiment.

Figure 11 shows a Finite Element model of the whole system. This model uses 94,877 ten-node quadratic elements (C3D10) and 512,346 degrees of freedom. Each mechanical part of the brake system is recalibrated with an experimental frequency response so that the deviation of the natural frequency component is lower than $2 \%$. The entire brake system model is also recalibrated for frequency. The contact law between the pads and the disc is exponential. This law was calibrated so that the FE model could reproduce the compressibility test (cf. Figure 6). The real normal modes of the FE model were computed in two steps in ABAQUS. First, the brake pressure and the rotational speed of the disc were simulated in a nonlinear static analysis to obtain the contact pressure field between the pads and the disk. Second, the stiffness was linearized in order to compute a real eigenvalue analysis.

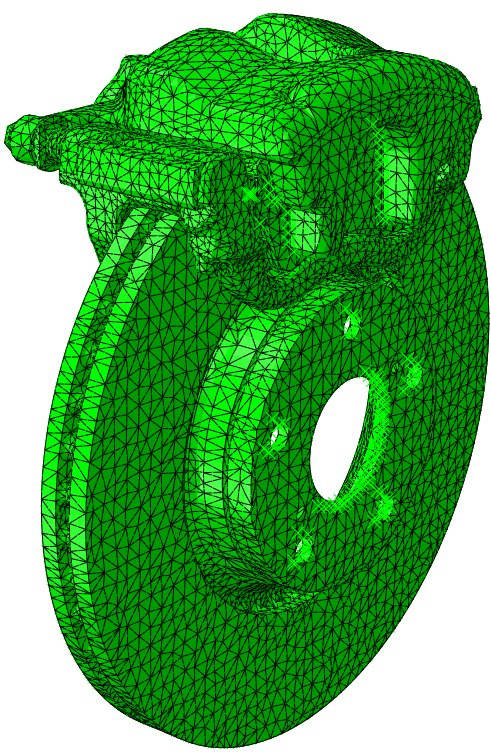

Figure 11: Finite Element model of the brake system.
The real eigenvectors of the FE model with a brake pressure of 3 bars were computed. These eigenvectors were restricted to the degrees of freedom corresponding to the sensor positions. Note that such restricted vectors no longer form a basis because they lose their orthonormality. The restricted eigenvectors $\varphi_{i}$ were then compared to the vectors of the pad motions observed $\psi_{j}=\left\{u_{n}\right\}_{j}$ using the MAC (cf. Equation (4)). Figure 12 shows the MAC of these eigenvectors with the pad motion at $1515 \mathrm{~Hz}$ between $t=3.6 \mathrm{~s}$ and $t=3.8 \mathrm{~s}$. No eigenvector of the FE model at 3 bar clearly corresponded to the pad motion observed just before the squeal noise occurred. The best MAC value was $57 \%$, obtained for mode 11. This mode had an eigenfrequency of $995 \mathrm{~Hz}$ far from the $1515 \mathrm{~Hz}$ of the pad motion observed. Although better MAC values could have been expected, several eigenvectors had a significant MAC value. Thus the pad motion observed could be represented with a linear combination of several restricted eigenvectors, for instance with modes 2, 8, 10, 11, 12 and 15 (cf. Figure 12).

The real eigenvectors of the FE model with a brake pressure of 5 bars were computed. Figure 13 shows the MACs of these eigenvectors with the pad motions at $1715 \mathrm{~Hz}$ and $3430 \mathrm{~Hz}$ between $t=6.0 \mathrm{~s}$ and $t=6.2 \mathrm{~s}$. No eigenvector of the FE model clearly corresponded to the pad motions observed during the occurrence of squeal noise. The best MAC for the fundamental frequency was $46 \%$, obtained for mode 16. The best MAC for the second harmonic frequency was $68 \%$, obtained for mode 5 . Once again, these modes had eigenfrequencies very different from the observations. Although better MAC values could have been expected, several eigenvectors had a significant MAC value. Thus the 


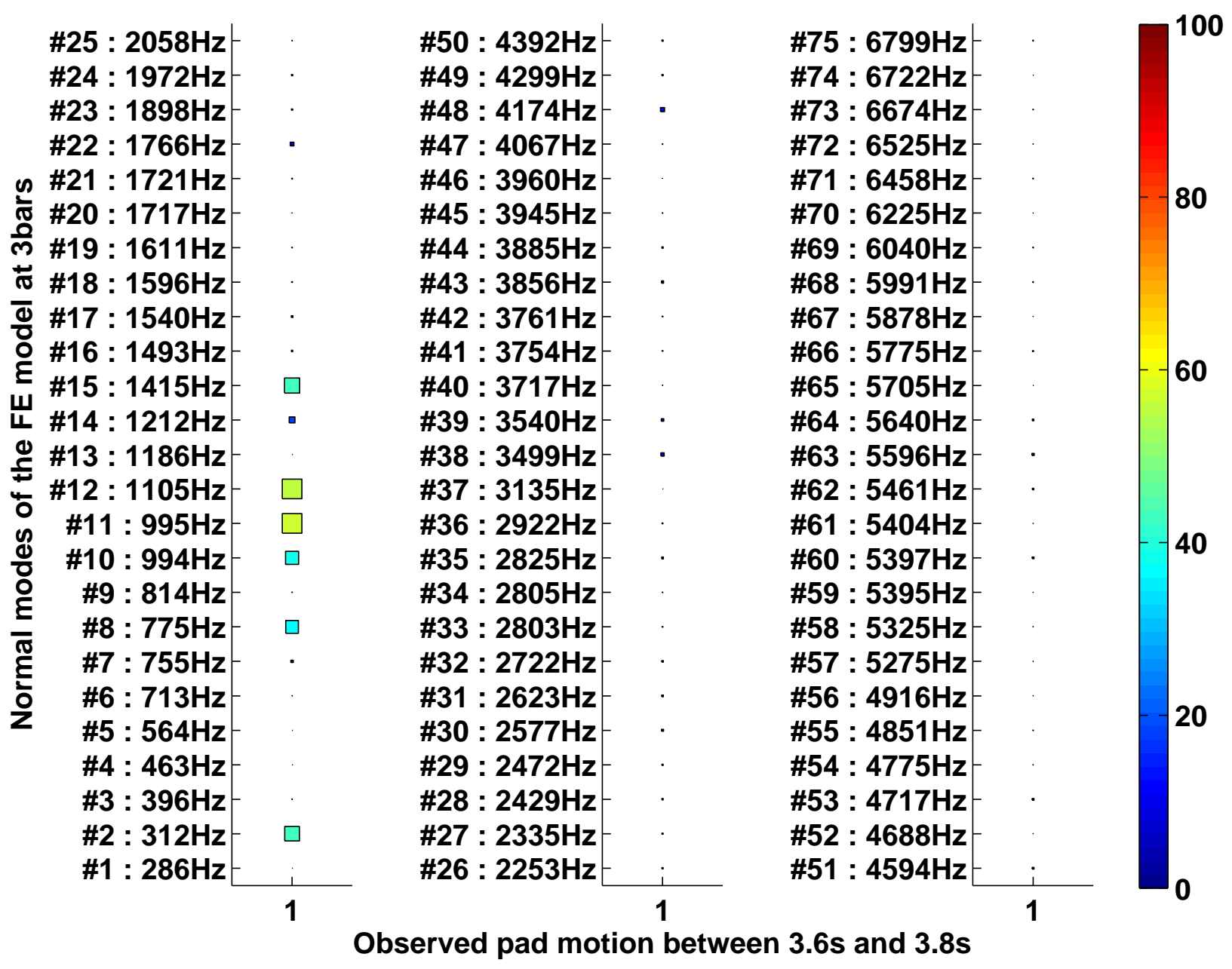

Figure 12: Before squeal occurrence, i.e. between 3.6s and 3.8s. Modal Assurance Criterion between the normal modes of the FE model at 3 bar and the pad motion observed. The colorbar represents the value of the MAC in $\%$. The fundamental frequency is $1515 \mathrm{~Hz}$ and denoted by 1 . The best MAC value is $57 \%$, is obtained for mode 11 .

pad motions observed could be represented with a linear combination of several restricted eigenvectors, for instance with modes 15, 16, 18, 19, 20, 22 and 26 for the fundamental frequency and with modes 5, 25, 32, 33, 35, 37 for the second harmonic (cf. Figure 12).

These deviations could have several explanations. First of all, the true pad topography was not taken into account, although this pad property is known to be of great importance (cf. AbuBakar and Ouyang [8]). Second, uncertainty in contact joints such as between the caliper and the bracket could have led to poor stiffness modelling of these contacts. Third, the effective brake pressure during the experiment was not known accurately. For example, the brake pressure between $t=3.6 \mathrm{~s}$ and $t=3.8 \mathrm{~s}$ was $3 \pm 0.5$ bar. Thus the FE model may not have accounted for the same brake pressure. Finally, although the mouvements observed were complex, the comparison was performed over a real modal basis and not over a complex modal basis. Thus these comparisons show that attention must be paid to the modelling of the effective contact surfaces. Although the MAC values were low, several eigenvectors had a significant MAC value. Thus pad motions should be represented with a linear combination of eigenvectors of real modal bases. Hence real modal bases seem suitable for model reduction before performing transient analysis or complex eigenvalue analysis. Moreover, before carrying out model reduction, Figures 12 and 13 could help in the selection of the eigenvectors to be kept in the basis for projection. 


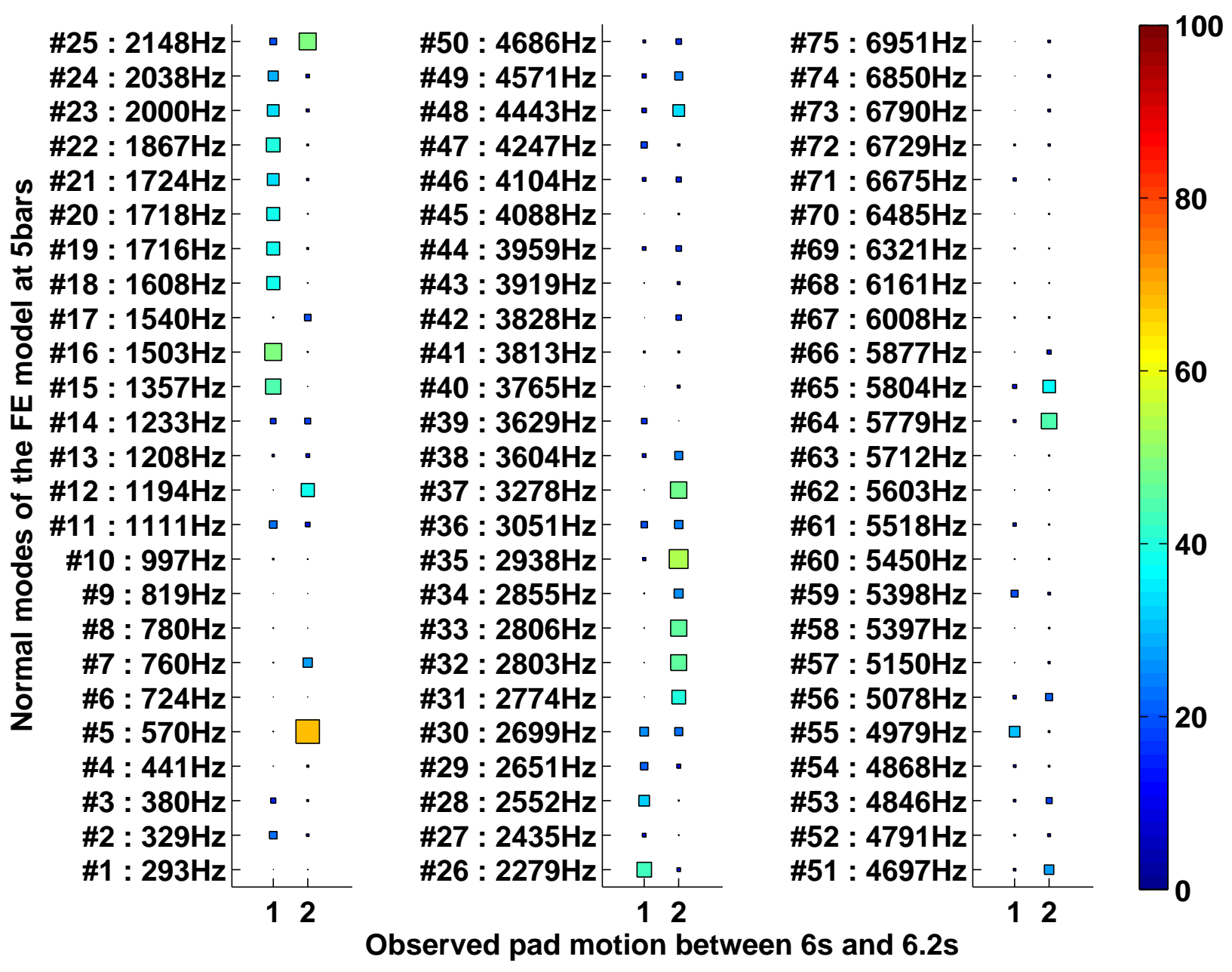

Figure 13: During the squeal occurrence, i.e. between 6.0s and 6.2s. The Modal Assurance Criterion between the normal modes of the FE model at 5 bars and the pad motion observed. The colorbar represents the value of the MAC in \%. The fundamental frequency is $1715 \mathrm{~Hz}$ and is denoted by 1 . Its best MAC value is $49 \%$, obtained for mode 16 . The second harmonic frequency is $3430 \mathrm{~Hz}$ denoted by 2 . Its best MAC value is $68 \%$, obtained for mode 5 .

\section{Conclusion}

Whereas many authors have studied the phenomenon of squeal noise on simplified test rigs, here, a squeal noise experiment was performed on a real brake system. As the authors expected brake pads to play a key role in squeal noise phenomena, six three-axis accelerometers were fixed on the backplate of the piston pad. The accelerations measured showed that the squeal frequency was dependent on brake pressure. The spectrum of the pad's acceleration presented a fundamental frequency and two harmonics during squeal noise occurrence. These harmonics highlighted the nonlinear nature of the squeal noise phenomenon. Although the squeal fundamental frequency was present almost throughout the experiment, squeal noise was only audible when the harmonics emerged.

Here, squeal could not be attributed to a stick-slip phenomenon. The authors presume that the nonlinear contact stiffness between pad and disc is a possible explanation for the harmonic rates observed. This hypothesis could be verified in future work by measuring the relative displacements between the pad and the other parts.

The backplate motion of the piston pad was interpolated using the Finite Element method applied to acceleration measurements. A frequential component was present in the acceleration spectrum before squeal occurs. This 
frequential component became the fundamental frequency during the squeal event. The pad motion associated with this frequential component was the same bending motion before and during the squeal event. Moreover harmonic components occurred along with the occurrence of squeal noise. The pad motion associated with the second harmonic was composed of a torsion plus a bending motion mostly involving the leading edge of the pad.

A detailed FE model was used. It was recalibrated with the experimental frequency response of each individual part and of the whole brake system, and with the contact pressure law of the pad. This model was used to compute a real modal basis. The eigenvectors of this basis were compared to the motions observed using the Modal Assurance Criterion. Since no eigenvector clearly corresponded to the motion observed, this comparison showed that attention must be paid to the modelling of effective contact surfaces. Although better MAC values could have been expected, several eigenvectors had a significant MAC value. Hence the motions observed could be represented by a linear combination of several eigenvectors of the basis.

To improve our knowledge still further, it would be interesting to perform an experimental investigation on the influence of the coincidence between a harmonic and an unstable mode.

\section{Acknowledgements}

This measurement campaign was performed thanks to the financial and technical help of the Robert BOSCH Foundation.

\section{References}

[1] O. Giannini, A. Akay, F. Massi, Experimental analysis of brake squeal noise on a laboratory brake setup, Journal of Sound and Vibration 292 (2006) $1-20$.

[2] O. Giannini, A. Sestieri, Predictive model of squeal noise occurring on a laboratory brake, Journal of Sound and Vibration 296 (2006) 583 601.

[3] O. Giannini, F. Massi, Characterization of the high-frequency squeal on a laboratory brake setup, Journal of Sound and Vibration 310 (2008) $394-408$.

[4] A. Akay, O. Giannini, F. Massi, A. Sestieri, Disc brake squeal characterization through simplified test rigs, Mechanical Systems and Signal Processing 23 (2009) 2590-2607.

[5] T. Butlin, J. Woodhouse, A systematic experimental study of squeal initiation, Journal of Sound and Vibration 330 (2011) $5077-5095$.

[6] P. Duffour, J. Woodhouse, Instability of systems with a frictional point contact - part 3: Experimental tests, Journal of Sound and Vibration 304 (2007) 186-200.

[7] A. R. AbuBakar, H. Ouyang, M. K. A. Hamid, A new prediction methodology for dynamic contact pressure distribution in a disc brake, Jurnal Teknologi 45(A) (2006) 1-11.

[8] A. R. AbuBakar, H. Ouyang, A prediction methodology of disk brake squeal using complex eigenvalue analysis., International Journal of Vehicle Design 46 (2008) 416-435.

[9] A. R. AbuBakar, H. Ouyang, Complex eigenvalue analysis and dynamic transient analysis in predicting disc brake squeal, International Journal of Vehicle Noise and Vibration 2 (2006) 143-155.

[10] C. L. Saw, C. G. Choong, A. R. Abu Bakar, M. R. Jamaluddin, W. M. M. W. Harujan, B. A. Ghani, Disc brake squeal suppression through chamfered and slotted pad, International Journal of Vehicle Structures and Systems 3 (2011) 28-35.

[11] W. Liu, G. M. Vyletel, J. Li, A rapid design tool and methodology for reducing high frequency brake squeal, in: Proceedings of the 24th Annual Brake Colloquim \& Exhibition.

[12] X. Lorang, F. Foymargiocchi, Q. Nguyen, P. Gautier, TGV disc brake squeal, Journal of Sound and Vibration 293 (2006) $735-746$.

[13] J. D. Fieldhouse, T. P. Newcomb, Double pulsed holography used to investigate noisy brakes, Optics and Lasers in Engineering 25 (1996) 455 - 494. Optical Diagnostics in the Automotive Industry.

[14] G. Dhatt, G. Touzot, E. Lefrançois, Méthode des éléments finis, Lavoisier, 2005.

[15] R. J. Allemang, The modal assurance criterion (mac): Twenty years of use and abuse, Sound and Vibration August (2003) 14-21. University of Cincinnati. 